Silva, MDG, Chavarette, FR \& Lourenço, FRB (2020). Analysis of the dynamic behavior of a mechanical system subject to unstable bifurcation. Research, Society and Development, 9(7): 1-26, e424973763.

\title{
Análise do comportamento dinâmico de um sistema mecânico sujeito a bifurcação instável
}

Analysis of the dynamic behavior of a mechanical system subject to unstable bifurcation Análisis del comportamiento dinámico de un sistema mecánico sujeto a bifurcación inestable

Recebido: 18/04/2020 | Revisado: 28/04/2020 | Aceito: 11/05/2020 | Publicado: 20/05/2020

Michael Dowglas de Gois Silva

ORCID: http://orcid.org/0000-0001-5162-9368

Universidade Estadual Paulista, Brasil

E-mail: michael@unirv.edu.br

Fabio Roberto Chavarette

ORCID: http://orcid.org/0000-0002-1203-7586

Universidade Estadual Paulista, Brasil

E-mail: fabio.chavarette@unesp.br

Rodrigo Francisco Borges Lourenço

ORCID: http://orcid.org/0000-0002-7772-6438

Universidade Estadual Paulista, Brasil

E-mail: rodrigoborges@unirv.edu.br

\section{Resumo}

Os sistemas estruturais esbeltos, susceptíveis a bifurcação instável, geralmente perdem a estabilidade para níveis de carga mais baixos do que a carga de flambagem linear da estrutura perfeita. No presente trabalho são estudadas as diversas configurações de equilíbrio dinâmico nas oscilações não lineares por meio de um sistema estrutural simples dado por um modelo barra rígida-mola com um grau de liberdade. A finalidade desse trabalho é estudar as diferentes bifurcações e oscilações não lineares por meio de uma análise paramétrica de um sistema estrutural simples sujeito a flambagem quando submetido a cargas compressivas. Para a resolução dos cálculos foi utilizado programas computacionais como o Maple, Matlab, Visual Studio $(\mathrm{C}++)$, assim como o Grapher para obtenção dos gráficos. Para obter as 
respostas estocásticas do modelo estudado será utilizado o polinômio de Legendre-Caos. Serão estudadas duas particularidades que são os sistemas que apresentam bifurcação simétrica do tipo Butterfly e os sistemas que apresentam bifurcação assimétrica do tipo Swallowtail, em ambos os casos as bifurcações apresentam um caminho pós-crítico inicial instável. Assim como a bifurcação do tipo Butterfly, a Swallowtail também é afetada de forma significativa em relação a presença de incertezas na rigidez do sistema. Dependendo do valor que se insere a incerteza, pode acontecer um aumento no número de soluções estáveis, mas dependendo do valor também pode gerar soluções caóticas. Independentemente da bifurcação analisada, os resultados obtidos se comportaram como a média dos resultados limites apenas para pequenos valores de $\Gamma_{1}$ ou para as soluções presentes no vale potencial pré-flambagem.

Palavras-chave: Flambagem; Bifurcação; Polinômio de caos; Fator de integridade.

\section{Abstract}

Slender structural systems, susceptible to unstable bifurcation, generally lose stability at lower load levels than the linear buckling load of the perfect structure. In the present work, the different dynamic balance configurations in nonlinear oscillations are studied through a simple structural system given by a rigid-spring bar model with a degree of freedom. The purpose of this work is to study the different bifurcations and nonlinear oscillations through a parametric analysis of a simple structural system subject to buckling when subjected to compressive loads. To solve the calculations, computer programs such as Maple, Matlab, Visual Studio $(\mathrm{C}++)$ were used, as well as Grapher to obtain the graphics. To obtain the stochastic responses of the studied model, the Legendre-Chaos polynomial will be used. Two particularities will be studied, which are the systems that present symmetrical bifurcation of the Butterfly type and the systems that present asymmetric bifurcation of the Swallowtail type, in both cases the bifurcations present an unstable initial post-critical path. Like the Butterfly-type bifurcation, Swallowtail is also significantly affected by the presence of uncertainties in the system's stiffness. Depending on the value that the uncertainty is inserted, an increase in the number of stable solutions can happen, but depending on the value it can also generate chaotic solutions. Regardless of the bifurcation analyzed, the results obtained behaved as the average of the limit results only for small values of $\Gamma 1$ or for the solutions present in the pre-buckling potential valley.

Keywords: Buckling; Bifurcation; Chaos polynomial; Integrity factor. 


\section{Resumen}

Los sistemas estructurales delgados, susceptibles a la bifurcación inestable, generalmente pierden estabilidad a niveles de carga más bajos que la carga de pandeo lineal de la estructura perfecta. En el presente trabajo, las diversas configuraciones de equilibrio dinámico en oscilaciones no lineales se estudian a través de un sistema estructural simple dado por un modelo de barra de resorte rígido con cierto grado de libertad. El propósito de este trabajo es estudiar las diferentes bifurcaciones y oscilaciones no lineales a través de un análisis paramétrico de un sistema estructural simple sujeto a pandeo cuando se somete a cargas de compresión. Para resolver los cálculos, se utilizaron programas informáticos como Maple, Matlab, Visual Studio $(\mathrm{C}++)$, así como Grapher para obtener los gráficos. Para obtener las respuestas estocásticas del modelo estudiado, se utilizará el polinomio Legendre-Chaos. Se estudiarán dos particularidades, que son los sistemas que presentan la bifurcación simétrica del tipo Mariposa y los sistemas que presentan la bifurcación asimétrica del tipo Swallowtail, en ambos casos las bifurcaciones presentan una ruta poscrítica inicial inestable. Al igual que la bifurcación tipo mariposa, Swallowtail también se ve significativamente afectado por la presencia de incertidumbres en la rigidez del sistema. Dependiendo del valor que se inserte la incertidumbre, puede ocurrir un aumento en el número de soluciones estables, pero dependiendo del valor también puede generar soluciones caóticas. Independientemente de la bifurcación analizada, los resultados obtenidos se comportaron como el promedio de los resultados límite solo para valores pequeños de $\Gamma 1$ o para las soluciones presentes en el potencial valle previo al pandeo.

Palabras clave: Pandeo; Bifurcación; Polinomio del caos; Factor de integridad.

\section{Introdução}

Nos últimos anos engenheiros e pesquisadores têm sido solicitados, cada vez com mais frequência, a estudar e a projetar estruturas com um grau maior de complexidade. Com isso os efeitos não lineares se tornam cada vez maiores e difíceis de serem controlados. Fenômenos não lineares são, entretanto, indesejáveis. Evitam-se estes tipos de problemas projetando-se estruturas de tal modo que elas trabalhem em regime linear, isso porque tanto a análise estática quanto a dinâmica de sistemas não lineares apresentam nas áreas de matemática e mecânica aplicada uma variedade de problemas de difícil solução.

Millon (1991) estudou a correlação entre as bifurcações e as oscilações não lineares de um sistema mecânico discreto com diferentes comportamentos pós-críticos, através de uma 
detalhada análise paramétrica. Para os vários tipos de bifurcação estudou-se, como o comportamento dinâmico varia com o nível de carregamento estático, com os parâmetros que caracterizam o carregamento dinâmico e com os parâmetros que caracterizam a rigidez do sistema estrutural. Neste trabalho, tem-se o mesmo modelo mecânico apresentado em Millon (1991), que é uma adaptação de um modelo barra rígida-mola, já utilizado anteriormente no estudo da estabilidade elástica de sistemas estáticos.

Segundo Millon (1991) diversos elementos estruturais, tais como colunas, pórticos, cascas, placas e arcos, dentre outros, quando submetidos a cargas compressivas, estão sujeitos ao fenômeno de flambagem, quer através de bifurcações, quer através da existência de pontos limites ao longo de um caminho de equilíbrio não linear. Millon (1991) analisa os três pontos de bifurcações mais comuns em sistemas estruturais: bifurcação simétrica estável, bifurcação simétrica instável e bifurcação assimétrica, dando especial atenção a duas particularidades da bifurcação: a simétrica instável e a bifurcação assimétrica que são, respectivamente, denominadas como bifurcações do tipo Butterfly e Swallowtail. O estudo das bifurcações é importante, pois fornecem modelos de transições e instabilidades quando alguns parâmetros são variados (Nayfeh \& Balachandran, 1995; Cedolin \& Bazant, 2003; Hoff, 2014).

A avaliação da segurança e da integridade de um sistema dinâmico não-linear é assunto de muita importância em engenharia e não depende exclusivamente da estabilidade das soluções pós-crítica. Um método para se estudar a integridade de um sistema dinâmico é através da análise da evolução das bacias de atração de várias soluções. Essa questão foi abordada pela primeira vez por Thompson (1989) e tem recebido atenção recentemente para a determinação de duas importantes medidas de segurança das bacias de atração: o fator de integridade local (FIL) e o fator de integridade global (FIG), que medem a máxima região segura e contínua da bacia de atração em torno de um dos atratores da resposta dinâmica (Rega e Lenci, 2005). Essas medidas de segurança têm sido aplicadas a diversos sistemas mecânicos ou contínuos (Lenci \& Rega, 2003; Rega \& Lenci, 2008; Gonçalves, Silva, Rega \& Lenci, 2011; Silva \& Gonçalves, 2011)

Silva \& Gonçalves (2011) investigaram a influência das incertezas dos sistemas reais e ruído da carga dinâmica na integridade e estabilidade das suas soluções em um sistema dinâmico. Para ilustrar a sensibilidade do sistema, um modelo discreto de sistemas, susceptível a bifurcação instável, é usado. É dada especial atenção à influência de incertezas e do ruído aleatório sobre as bacias de atração do sistema e, consequentemente, nas medidas de integridade do sistema forçada e não forçado. 
Seguindo a mesma linha de pesquisa Silva, Brazão \& Gonçalves (2015) investigaram a influência de incertezas nos parâmetros físicos e geométricos para a determinação da carga de instabilidade paramétrica de uma casca cilíndrica excitada axialmente, utilizando o método de Galerkin Estocástico juntamente com o polinômio de Hermite-Caos. A incerteza é considerada inicialmente em apenas um de seus parâmetros: no módulo de elasticidade, na espessura e na amplitude da imperfeição geométrica inicial. Em seguida é analisado a influência de aleatoriedades em dois parâmetros simultaneamente, sendo eles: a espessura e o módulo de elasticidade. Diferentemente de Silva \& Gonçalves (2011), a incerteza foi considerada como uma função de densidade de probabilidade definida e as incertezas são incorporadas diretamente como um parâmetro da eq. diferencial de equilíbrio.

O objetivo deste trabalho é estudar as diferentes bifurcações e oscilações não lineares através de uma análise paramétrica de um sistema estrutural simples sujeito a flambagem quando submetido a cargas compressivas. Estas estruturas quando carregadas, na presença de perturbações ou carga dinâmicas, podem apresentar um comportamento dinâmico bastante complexo, tais como oscilações periódicas e caóticas. Visando isso, se objetiva a avaliação da influência das incertezas dos parâmetros físicos e geométricos nas vibrações não-lineares e na estabilidade desse sistema mecânico sujeito a flambagem, bem como a aplicabilidade do polinômio de caos generalizado para o estudo das aleatoriedades deste sistema.

\section{Metodologia}

Para o desenvolvimento desse projeto será utilizado uma revisão aprofundada da literatura sobre os diferentes mecanismos de perda de estabilidade em sistemas discretos, através de pesquisa em livros, internet, artigos, teses e dissertações da área. Para a resolução dos cálculos foi utilizado programas computacionais como o Maple, Matlab, Visual Studio $(C++)$, assim como o Grapher para obtenção dos gráficos. Assim como a implementação de códigos já existentes.

O modelo representado na Figura 1 consiste numa barra rígida de peso desprezível, comprimento $L$, presa por duas molas: uma mola vertical de rigidez linear $k_{1}$ e uma mola de rigidez $\bar{k}_{2}$ que pode ser linear se $\eta$ for igual a zero e não linear caso $\eta$ seja igual a um, (ver eq.(1)) e uma massa concentrada $M$ fixa no ponto $A$. Segundo Millon (1991) modelos semelhantes a esse têm sido frequentemente usados para representar e estudar de maneira 
simplificada o comportamento de elementos estruturais tais como colunas, arcos, cascas e placas.

Figura 1 - Sistema massa-mola-barra rígida com um grau de liberdade: (a) Estático (b) Dinâmico.

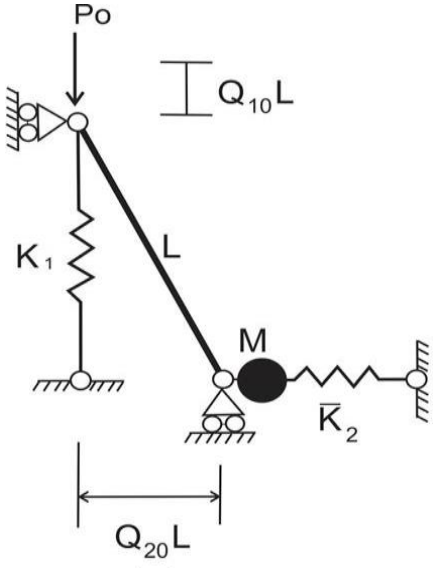

(a)

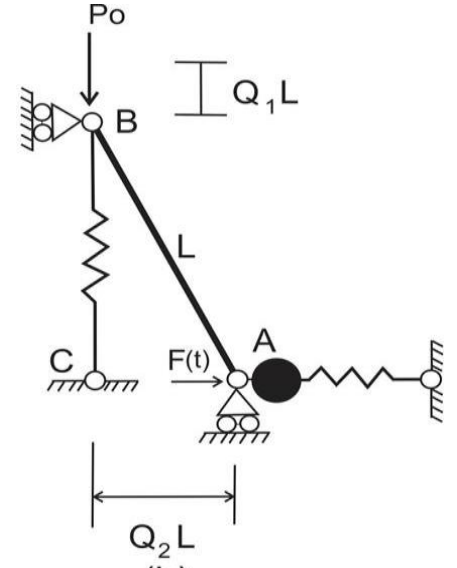

(b)

Fonte: Adaptado Millon, 1991.

A formulação aqui proposta segue a formulação apresentada por Millon (1991), sendo os principais passos mostrados a seguir.

A rigidez $\bar{k}_{2}$ é representada pela seguinte eq.:

$\bar{k}_{2}=k 2\left\lfloor 1+\eta\left(\alpha Q_{20}+\beta Q_{20}{ }^{2}+\gamma Q_{20}{ }^{3}+\delta Q_{20}{ }^{4}\right)\right]$

Esta equação será utilizada para exemplificar os vários tipos de comportamento póscrítico que ocorrem em vários sistemas estruturais. Serão estudados dois casos específicos: Butterfly e Swallowtail, que possuem parâmetros diferentes que regem a não linearidade da mola $\bar{k}_{2}$.

A equação da variação da energia potencial total do sistema é dada da seguinte forma:

$$
\begin{aligned}
& \Delta \Pi=\Delta U-\Delta W e=\frac{1}{2} k_{1} L^{2}\left[\left(1-\sqrt{1-Q_{2 t}{ }^{2}}\right)^{2}-\left(1-\sqrt{1-Q_{20}{ }^{2}}\right)^{2}\right] \\
& +\frac{1}{2} k_{2} L^{2}\left[1+\eta\left(\frac{2}{3} \alpha Q_{2 t}+\frac{2}{4} \beta Q_{2 t}{ }^{2}+\frac{2}{5} \gamma Q_{2 t}{ }^{3}+\frac{2}{6} \delta Q_{2 t}{ }^{4}\right)\right] Q_{2 t}{ }^{2} \\
& -\frac{1}{2} k_{2} L^{2}\left[1+\eta\left(\frac{2}{3} \alpha Q_{20}+\frac{2}{4} \beta Q_{20}{ }^{2}+\frac{2}{5} \gamma Q_{20}{ }^{3}+\frac{2}{6} \delta Q_{20}{ }^{4}\right)\right] Q_{20}{ }^{2} \\
& -\overline{P_{0}} L\left(1-\sqrt{1-Q_{2 t}{ }^{2}}\right)+\overline{P_{0}} L\left(1-\sqrt{1-Q_{20}{ }^{2}}\right)-F(t) Q_{2} L
\end{aligned}
$$


onde $Q_{2 t}=Q_{2}(t)+Q_{20}$ representa o deslocamento total do sistema.

A variação da energia cinética é obtida por:

$\Delta T=\frac{1}{2} M v^{2}$

Sendo:

$v=\frac{d \Delta h_{t}}{d Q_{2 t}} \frac{d Q_{2 t}}{d t}$

Através das eqs. (2) e (3) obtêm-se a função de Lagrange:

$$
\begin{aligned}
& \Delta L=\Delta T-\Delta \Pi=\frac{1}{2} M\left(\dot{Q}_{2} L\right)^{2}-\frac{1}{2} k_{1} L^{2}\left[\left(1-\sqrt{1-Q_{2 t}{ }^{2}}\right)^{2}-\left(1-\sqrt{1-Q_{20}{ }^{2}}\right)^{2}\right] \\
& -\frac{1}{2} k_{2} L^{2}\left[1+\eta\left(\frac{2}{3} \alpha Q_{2 t}+\frac{2}{4} \beta Q_{2 t}{ }^{2}+\frac{2}{5} \gamma Q_{2 t}{ }^{3}+\frac{2}{6} \delta Q_{2 t}{ }^{4}\right)\right] Q_{2 t}{ }^{2} \\
& +\frac{1}{2} k_{2} L^{2}\left[1+\eta\left(\frac{2}{3} \alpha Q_{20}+\frac{2}{4} \beta Q_{20}{ }^{2}+\frac{2}{5} \gamma Q_{20}{ }^{3}+\frac{2}{6} \delta Q_{20}{ }^{4}\right)\right] Q_{20}{ }^{2} \\
& -\overline{P_{0}} L\left(1-\sqrt{1-Q_{2 t}{ }^{2}}\right)+\overline{P_{0}} L\left(1-\sqrt{1-Q_{20}{ }^{2}}\right)-F(t) Q_{2} L
\end{aligned}
$$

Atribuindo as seguintes variáveis auxiliares:

$\Omega_{n}{ }^{2}=\frac{k_{2}}{M} ; \lambda \Omega_{n}{ }^{2}=\frac{k_{1}}{k_{2}} ; 2 \xi \Omega_{n}=\frac{c}{L M} ; P_{0} \Omega_{n}{ }^{2}=\frac{\overline{P_{0}}}{L M} ; f(t) \Omega_{n}{ }^{2}=\frac{F(t)}{L M}+\frac{F_{0}}{L M}$

e colocando a equação em termos de um tempo $\tau$ adimensional, sendo $t=\frac{\tau}{\Omega_{n}}$, tem-se que:

$d \tau=\Omega_{n} d t$

$d \tau^{2}=\Omega_{n}{ }^{2} d^{2} t$

A partir da equação de Lagrange e da aplicação do princípio de Hamilton, obtém-se a seguinte equação de movimento do sistema:

$$
\begin{aligned}
& \frac{d^{2} Q_{2}}{d \tau^{2}}+2 \xi \frac{d Q_{2}}{d \tau}+\left[\lambda-P_{0}\right]\left(\frac{Q_{2 t}}{\sqrt{1-Q_{2 t}{ }^{2}}}\right)+[1-\lambda] Q_{2 t}+ \\
& \eta\left(\alpha Q_{2 t}{ }^{2}+\beta Q_{2 t}{ }^{3}+\gamma Q_{2 t}{ }^{4}+\delta Q_{2 t}{ }^{5}\right)=f(\tau)
\end{aligned}
$$

Neste trabalho, a não linearidade ocorre devido a presença da mola não linear $\bar{k}_{2}$, eq. (1), permitindo a avaliação da influência das incertezas dos parâmetros geométricos, 
representados na eq. (1) por: $\alpha, \beta, \delta e \gamma$, nas vibrações não lineares e na estabilidade do modelo apresentado na Figura 1. As incertezas serão consideradas com uma função de distribuição de probabilidade uniforme, dessa forma, o polinômio de Legendre-Caos é indicado na solução dos problemas estocásticos via polinômio de caos (Xiu \& Karniadakis, 2002).

Os polinômios de caos generalizados, segundo Xiu \& Karniadakis (2002), aproximam um processo estocástico através de polinômios ortogonais de variáveis aleatórias, sendo representados por processos estocásticos de segunda ordem $X(t, x ; \phi)$ :

$$
\begin{aligned}
X(t, x ; \phi) & =a_{0}(x, t) \vartheta_{0}+\sum_{i_{1}=1}^{\infty} a_{i_{1}}(x, t) \vartheta_{1}\left(\xi_{i_{1}}(\phi)\right)+\sum_{i_{1}=1}^{\infty} \sum_{i_{2}=1}^{i_{1}} a_{i_{1} i_{2}}(x, t) \vartheta_{2}\left(\xi_{i_{1}}(\phi), \xi_{i_{2}}(\phi)\right) \\
& +\sum_{i_{1}=1}^{\infty} \sum_{i_{2}=1}^{i_{1}} \sum_{i_{3}=1}^{i_{2}} a_{i_{1} i_{2} i_{3}}(x, t) \vartheta_{3}\left(\xi_{i_{1}}(\phi), \xi_{i_{2}}(\phi), \xi_{i_{3}}(\phi)\right)+\cdots
\end{aligned}
$$

onde $\phi$ é um evento randômico, $t$ é o tempo, $x$ é o espaço e $\vartheta_{0}\left(\xi_{i_{1}}, \ldots, \xi_{i_{n}}\right)$ denota o polinômio de caos generalizado de grau $n$ nas variáveis $\left(\xi_{i_{1}}, \ldots, \xi_{i_{n}}\right)$. Tais polinômios são ortogonais em termos da variável aleatória multidimensional $\xi=\left(\xi_{i_{1}}, \ldots, \xi_{i_{n}}\right)$, com $\vartheta_{0}=1$. Por conveniência, reescreve-se a eq. (9) da seguinte forma:

$$
X(t, x ; \phi)=\sum_{i=0}^{\infty} \hat{a}_{i}(x, t) \Phi_{i}(\xi)
$$

onde existe uma correspondência um para um entre as funções $\vartheta_{n}\left(\xi_{i_{1}}, \ldots, \xi_{i_{n}}\right)$ e $\Phi_{i}(\xi)$ bem como entre seus respectivos coeficientes $a_{i_{1} i_{2} i_{3}}$ e $\hat{a}_{i}$. Os polinômios ortogonais satisfazem a seguinte relação de ortogonalidade:

$$
\left\langle\Phi_{i} \Phi_{j}\right\rangle=\left\langle\Phi_{i}^{2}\right\rangle \delta_{i j}
$$

onde $\delta_{i j}$ é a função delta de Kronecker e $\left\langle\Phi_{i}^{2}\right\rangle$ é o produto interno entre as funções. Por definição, o produto interno entre polinômios ortogonais, para um caso contínuo, é dado por:

$$
\langle f(\xi), g(\xi)\rangle=\int f(\xi) g(\xi) w(\xi) d(\xi)
$$

onde $w(\xi)$ denota a função densidade de probabilidade e o intervalo de integração é o domínio onde a variável aleatória é definida. 
Em termos práticos, a eq. (10) divide o campo estocástico $X(t, x ; \phi)$ em uma parte determinística que é a parcela $\hat{a}_{i}(x, t)$ e uma parte estocástica dado pelo polinômio caos $\Phi_{i}(\xi)$. As eqs. (9) e (10) são truncadas em um número finito de termos, $P$, onde a variável aleatória $\xi$ é um vetor aleatório $n$-dimensional.

$$
X(t, x ; \phi)=\sum_{i=0}^{P} \hat{a}_{i}(x, t) \Phi_{i}(\xi)
$$

O número total de termos de uma expansão de polinômios caos é $P+1$, obtido pela dimensionalidade da variável aleatória $\xi(n)$ e pela ordem da expansão polinomial desejada.

Neste trabalho será usado o polinômio de Legendre-Caos, para o caso contínuo, com as variáveis aleatórias do tipo uniforme, num intervalo $a \leq x \leq \mathrm{b}$ específico, que será definido através da variável onde se deseja avaliar a incerteza. Os polinômios de Legendre-Caos, denotados por $\left\{\operatorname{Le}_{\mathrm{n}}(x)\right\}, n=0,1,2, \ldots$, para um domínio $[a, b]$ possuem a função densidade de probabilidade igual a:

$$
w(\xi)=\frac{1}{b-a}
$$

A seguir, estão representados os setes primeiros polinômios de Legendre-Caos definido no intervalo de $[a, b]=[-1,1]$ :

$$
\begin{aligned}
& L e_{0}=1 \\
& L e_{1}=x \\
& L e_{2}=\frac{1}{2}\left(3 x^{2}-1\right) \\
& L e_{3}=\frac{1}{2}\left(5 x^{3}-3 x\right) \\
& L e_{4}=\frac{1}{8}\left(35 x^{4}-30 x+3\right) \\
& L e_{5}=\frac{1}{8}\left(63 x^{5}-70 x^{3}+15 x\right) \\
& L e_{6}=\frac{1}{16}\left(231 x^{6}-315 x^{4}+105 x^{2}-5\right)
\end{aligned}
$$

Adotando na eq. (8) que:

$$
f(\tau)=\Gamma_{1} \cos (\Omega \tau)
$$


onde $\Gamma_{1}$ é a amplitude da carga de excitação e $\Omega$ o parâmetro de frequência de excitação. Substituindo a eq. (16) na eq. (8) tem-se que:

$$
\begin{aligned}
& \frac{d^{2} Q_{2}}{d \tau^{2}}+2 \xi \frac{d Q_{2}}{d \tau}+\left[\lambda-\overline{P_{0}}\right]\left(\frac{Q_{2}}{\sqrt{1-Q_{2}{ }^{2}}}\right)+[1-\lambda] Q_{2} \\
& +\eta\left(\alpha Q_{2}{ }^{2}+\beta Q_{2}{ }^{3}+\gamma Q_{2}{ }^{4}+\delta Q_{2}{ }^{5}\right)-\Gamma_{1} \cdot \cos (\Omega \cdot \tau)=0
\end{aligned}
$$

As equações diferenciais ordinárias estocásticas (EDOE) são obtidas substituindo-se a incerteza $\phi$ no parâmetro desejado da eq. (17). Neste trabalho serão avaliados os parâmetros $\beta, \delta$ e $\alpha$. Após a obtenção da EDOE, aplica-se o método do polinômio de caos para obter-se as equações determinísticas equivalentes que consideram as incertezas do parâmetro no problema, a partir da eq. (12), permitindo a obtenção de informações estatísticas do problema como média e variância. A quantidade de equações obtidas será igual ao número de polinômios de Legendre-Caos usados para a formulação. Segundo Xiu \& Karniadakis (2002), o valor que acompanha o polinômio de primeira ordem corresponde à média e soma dos demais valores que acompanham os demais polinômios correspondentes à variância.

\section{Resultados e Discussão}

Para se estudar a classe de bifurcação Butterfly, simplifica-se a eq. (17) adotando os seguintes parâmetros: $\lambda=4, \beta=-6, \delta=4, \alpha=0$ e $\gamma=0$. Já para o estudo da classe de bifurcação Swallowtail é necessário tornar o termo $\alpha$ diferente de zero, para isto a eq. (17) passa a ter os seguintes parâmetros: $\lambda=4, \beta=0, \delta=0, \alpha=-3$ e $\gamma=0$. Nos dois casos de bifurcação avaliados neste trabalho, consideram-se as incertezas na ordem de $10 \%$ nos valores dos parâmetros determinísticos, ou seja, na bifurcação do tipo Butterfly serão considerados incertezas em $\beta$ e $\delta$, já para a bifurcação do tipo Swallowtail no parâmetro $\alpha$.

Para o traçado dos diagramas de bifurcação foi utilizado o método da força bruta, integrando-se a equação de equilíbrio (17), ao longo do tempo, a partir do método de RungeKutta de quarta ordem, e mapeando as seções de Poincarè obtidas no estado permanente da resposta no tempo. Para cada valor da amplitude de excitação da carga dinâmica $\left(\Gamma_{1}\right)$, assumido como parâmetro de controle, e para o parâmetro de frequência de excitação, $\Omega=$ 1.1, foram obtidas as seções de Poincarè e construídos os diagramas de bifurcação, que relacionam os valores de $Q_{2}(\tau)$ com os valores de $\Gamma_{1}$, destacando que apenas as soluções de equilíbrio estáveis são obtidas com essa técnica. Os diagramas foram obtidos para pré- 
carregamento estático $\overline{P_{0}}=0.5$ e considerando uma taxa de amortecimento $\xi=0.1$ para as duas classes de bifurcação: Butterfly e Swallowtail.

A bifurcação do tipo Butterfly apresenta dois parâmetros determinísticos, $\beta$ e $\delta$, que serão analisados separadamente, para se poder avaliar a influência da incerteza de cada parâmetro no comportamento global do sistema. Para a análise do comportamento da incerteza no parâmetro $\beta$ no diagrama de bifurcação além da bifurcação para o parâmetro nominal, $\beta=-6$, foram obtidas também as bifurcações para as incertezas $\beta=-5.4$ e $\beta=-6.6$. Para permitir a comparação do comportamento entre as bifurcações com incerteza no parâmetro $\beta$ foi construída a Figura 2a com os valores limites da incerteza considerada em $\beta$. O diagrama de bifurcação da Figura 2 a para $\beta=-6$, possui grupos de soluções diferente à medida que se incrementa o valor de $\Gamma_{1}$. Para valores $\Gamma_{1}<0.18$, a bifurcação apresenta três soluções periódicas estáveis. Com valores de $\Gamma_{1}$ entre 0.18 e 0.36 existem duas soluções periódicas estáveis. Já para valores $\Gamma_{1}>0.36$ o diagrama de bifurcação apresenta apenas uma solução periódica estável.

Figura 2 - Diagrama de bifurcação do tipo Butterfly considerando as incertezas no parâmetro:(a) $\beta$ e (b) $\delta .(\Omega=1.1, \lambda=4$ e $\xi=0.1)$.

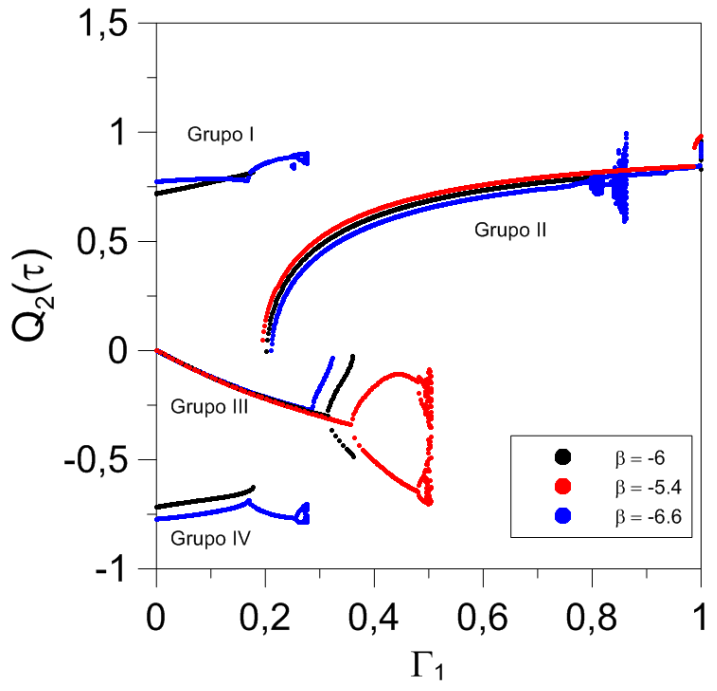

(a)

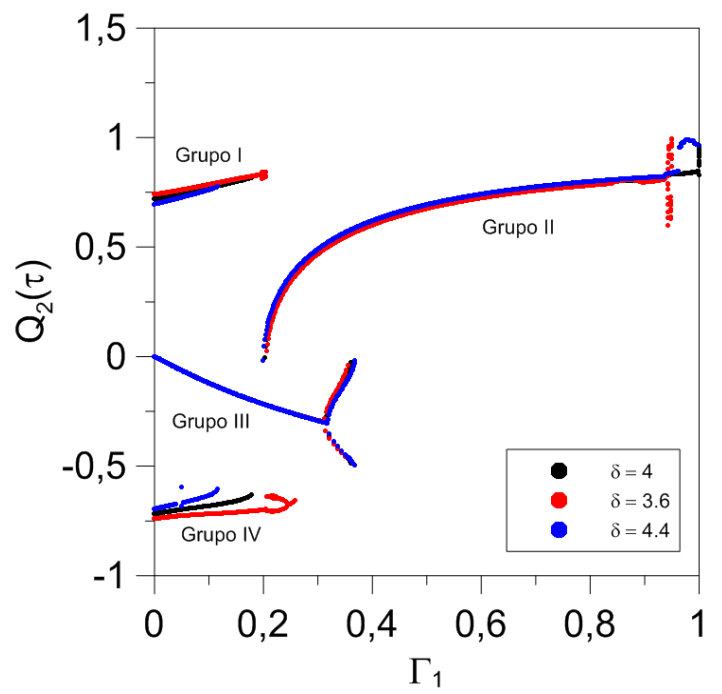

(b)

Fonte: Autores, 2020.

O diagrama de bifurcação para $\beta=-6.6$, representado pela cor azul na Figura 2a, apresenta uma distribuição de soluções estáveis análogas ao parâmetro nominal de $\beta$ (cor preta), com a redução de soluções estáveis à medida que se aumenta o valor de $\Gamma_{1}$. Mas essa 
incerteza apresenta uma particularidade para valores de $\Gamma_{1}$ entre 0.25 e 0.28 , que é a presença de quatro soluções periódicas estáveis, característica que não é apresentada por nenhuma outra bifurcação. Já a incerteza de $\beta=-5.4$, representado pela cor vermelha na Figura 2a, apresenta um número menor de soluções estáveis logo no início do diagrama de bifurcação, apresentando na maioria dos seus trechos somente uma solução periódica estável. Porém, ela apresenta uma particularidade, que é a presença de uma duplicação de soluções para $\Gamma_{1}$ entre 0.36 a 0.5 , obtendo assim nesse trecho três soluções periódicas estáveis para o mesmo valor de $\Gamma_{1}$, como ilustra a Figura 2a. A incerteza no parâmetro $\beta$ afeta de forma significativa o comportamento no diagrama de bifurcações da Figura 2a, fazendo surgir outras soluções periódicas estáveis para o mesmo valor de $\Gamma_{1}$.

Para a análise do comportamento da incerteza do parâmetro $\delta$ nos diagramas de bifurcações, assim como no caso de $\beta$, além da bifurcação para o parâmetro nominal, $\delta=4$, foram obtidas também as bifurcações para as incertezas $\delta=3.6$ e $\delta=4$.4. Para permitir a comparação do comportamento das bifurcações com incerteza em $\delta$ foi construída a Figura 2b. Diferentemente das bifurcações do tipo Butterfly apresentadas na Figura 2a com incerteza no parâmetro $\beta$, a Figura $2 \mathrm{~b}$ para o mesmo tipo de bifurcação, mas com a incerteza no parâmetro $\delta$, apresenta uma distribuição de soluções estáveis análoga entre as bifurcações com e sem a incerteza. A quantidade de soluções periódicas estáveis para o mesmo valor de $\Gamma_{1}$ são as mesmas, independentemente do incremento do valor de $\delta$. Para valores de $\Gamma_{1}<0.35$ se tem duas soluções periódicas estáveis, já para valores maiores que 0.35 existe apenas uma única solução periódica estável, como ilustrado na Figura 2b. A única particularidade aparece quando $\Gamma_{1}=0.19$, onde dependendo do valor de $\delta$ a bifurcação pode apresentar uma ou duas soluções periódicas estáveis.

Para a bifurcação do tipo Swallowtail existe apenas um parâmetro determinístico, $\alpha$, que será analisado para avaliar a influência da incerteza desse parâmetro no diagrama de bifurcação. Assim como na análise para o caso Butterfly, além da bifurcação para o parâmetro nominal, $\alpha=-3$, foram obtidas também os diagramas de bifurcação para as incertezas $\alpha=$ -2.7 e $\alpha=-3.3$. Para permitir a comparação do comportamento entre os diagramas de bifurcação com incerteza, construiu-se a Figura 3 com os casos limites do valor do parâmetro $\alpha$. O diagrama de bifurcação, para $\alpha=-3$, possui um traçado diferente, à medida que se incrementa o valor de $\Gamma_{1}$. Para valores de $\Gamma_{1}>0.27$, ou $0.15<\Gamma_{1}<0.19$, o diagrama de bifurcação apresenta apenas uma solução periódica estável. Para valores de $\Gamma_{1}<0.27$, para um mesmo valor de $\Gamma_{1}$ o diagrama de bifurcação apresenta duas soluções periódicas estáveis. 
O diagrama de bifurcação para $\alpha=-3.3$, representado pela cor azul na Figura 3, apresenta uma distribuição de soluções estáveis análoga ao diagrama de bifurcação com parâmetro nominal (cor preta), com a redução do número de soluções periódicas estáveis à medida que se aumenta o valor de $\Gamma_{1}$. Mas essa incerteza apresenta uma particularidade quando $\Gamma_{1}$ assume o valor de 0.32 que é a presença de três soluções periódicas estáveis, característica que não é apresentada por nenhuma das outras bifurcações apresentadas na Figura 3. Já o diagrama de bifurcação para $\alpha=-2.7$, dado pela cor vermelha na Figura 3, também apresenta um comportamento análogo ao diagrama de bifurcação com parâmetro nominal, apresentando, na maioria dos seus trechos, uma ou duas soluções estáveis à medida que se incrementa o valor de $\Gamma_{1}$. Porém, o diagrama de bifurcação apresenta uma particularidade, que é a presença de soluções caóticas para $0.15<\Gamma_{1}<0.19$. A incerteza no parâmetro $\alpha$ afeta de forma significativa o comportamento no diagrama de bifurcações da Figura 3, fazendo surgir outras soluções periódicas estáveis para o mesmo valor de $\Gamma_{1}$ e até soluções caóticas em determinados trechos.

Figura 3 - Diagrama de bifurcação do tipo Swallowtail considerando as incertezas no parâmetro $\alpha$. $(\Omega=1.1, \lambda=4$ e $\xi=0.1)$.

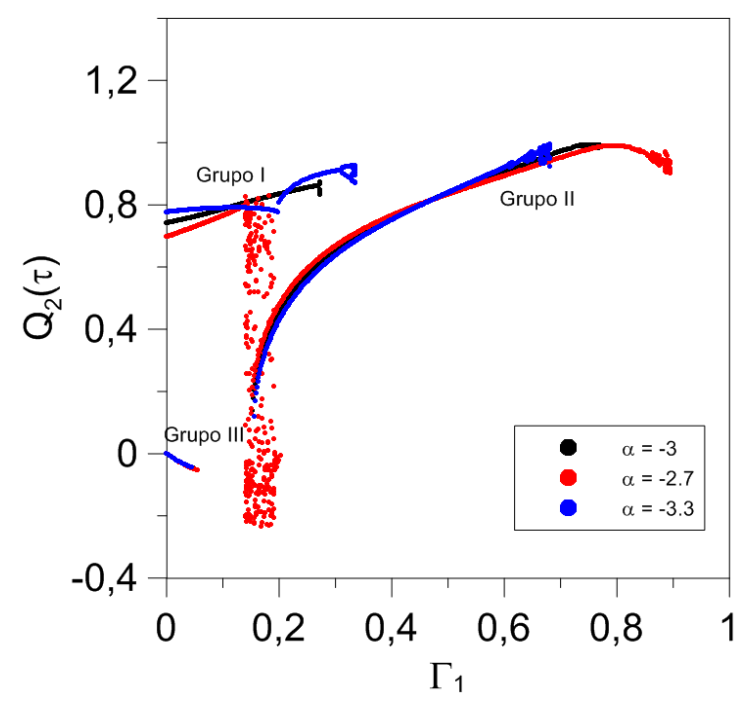

Fonte: Autores, 2020.

Assim como a bifurcação do tipo Butterfly, a Swallowtail também é afetada de forma significativa em relação a presença de incertezas na rigidez do sistema. Dependendo do valor que se insere a incerteza, pode acontecer um aumento no número de soluções estáveis, mas 
dependendo do valor também pode gerar soluções caóticas. A partir das Figs. 2 e 3 é possível afirmar que, independentemente da incerteza e do caso de bifurcação analisado, para valores de $\Gamma_{1}>0.5$ as soluções são estáveis, apresentando somente uma solução periódica. Pode-se notar também, que as incertezas afetam mais o comportamento dos diagramas de bifurcações para valores de $\Gamma_{1}<0.5$.

Para se avaliar a aleatoriedade dessas incertezas no sistema estudado, aplica-se o método do polinômio de caos, para obter-se as equações determinísticas equivalentes, a partir da eq. (12), que permitirá a obtenção de informações estatísticas do problema (média e variância) sem a necessidade de gerar amostras. Neste trabalho serão avaliados a aleatoriedade nos parâmetros $\beta, \delta$ e $\alpha$. Todos os diagramas de bifurcação deste trabalho que representam a variabilidade imposta pelas incertezas, obtidas pelo polinômio de Legendre-Caos, eq. (15), foram obtidas numericamente através da aplicação do método de Runge-Kutta de quarta ordem, incrementando-se a amplitude da excitação harmônica, $\Gamma_{1}$, e mantendo-se a frequência de excitação, $\Omega$, constante e igual 1.1 .

O incremento do número de polinômio de Legendre-Caos na solução do problema discretiza com mais precisão a função de densidade de probabilidade da variável aleatória, eq. (14), garantindo ao problema maior confiabilidade da cobertura do espectro amostral (Xiu; Karniadakis, 2002). Visando isso, será utilizado para análise das aleatoriedades dos três parâmetros $(\beta, \delta$ e $\alpha)$ os seis primeiros termos do polinômio de Legendre-Caos.

As Figs. 4a e 4b apresentam os diagramas de bifurcação do tipo Butterfly tanto para o caso determinísticos quanto para o não determinístico, cujo parâmetros que definem a rigidez do sistema são $\beta$ e $\delta$. O diagrama de bifurcação para o caso estocástico está representado pela cor verde nessas figuras. Já os diagramas de bifurcação para o parâmetro nominal e para as incertezas, estão representados pelas mesmas cores relacionadas anteriormente nas Figs. 2a e $2 b$.

De modo a comprovar a eficiência do método do polinômio de caos no estudo do problema não determinístico, comparam-se os diagramas de bifurcação determinístico para a bifurcação do tipo Butterfly com o diagrama obtido para o caso não determinístico (verde), considerando as mesmas condições de pré-carregamento. Para a validação do método é necessário que a bifurcação não determinística represente a variabilidade dos parâmetros $\beta \mathrm{e} \delta$ no intervalo $-6.6<\beta<-5.4$ e $3.6<\delta<4.4$, respectivamente.

Analisando as Figs. 4a e 4b, observa-se que os diagramas para o caso não determinístico se comportam como a média dos limites analisados no trecho inicial da bifurcação, quando $\Gamma_{1}$ 
$<0.32$. A medida que se incrementa $\Gamma_{1}$, as bifurcações têm um comportamento diferente do trecho inicial, compreendido ente as fronteiras das incertezas (azul e vermelha). Isso ocorre porque a técnica do polinômio de caos incorpora à análise do problema a variância das amostras na resposta no tempo do sistema, como ilustrado na Figura 5, que apresenta além da média do processo dado por $\mathrm{V}_{1}(\mathrm{t})$ a variância da resposta dada por $\mathrm{V}_{2}(\mathrm{t})$, obtida para a resposta estocástica da bifurcação do tipo Butterfly com incerteza em $\beta$.

Nas Figs. 5a e 5b, obtidas para o valor de $\Gamma_{1}=0.05$, observa-se que a variância é pequena levando a resposta não determinística do sistema a se aproximar da média aritmética. Já as Figs. 5 c e $5 d$, obtida para um valor de $\Gamma_{1}=0.33$, a variância é suficiente para modificar a resposta não determinística do sistema, levando a resposta não determinística divergir da média aritmética dos diagramas de bifurcação.

Figura 4 - Diagramas de bifurcação determinístico e não determinístico do tipo Butterfly: (a) com incerteza em $\beta$ e (b) com incerteza em $\delta .(\Omega=1.1, \lambda=4$ e $\xi=0.1)$.

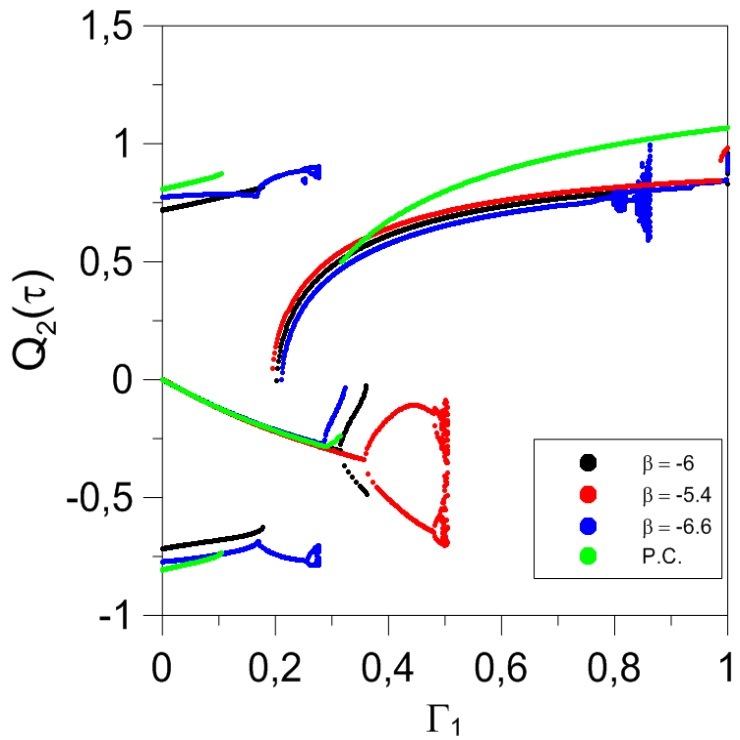

(a)

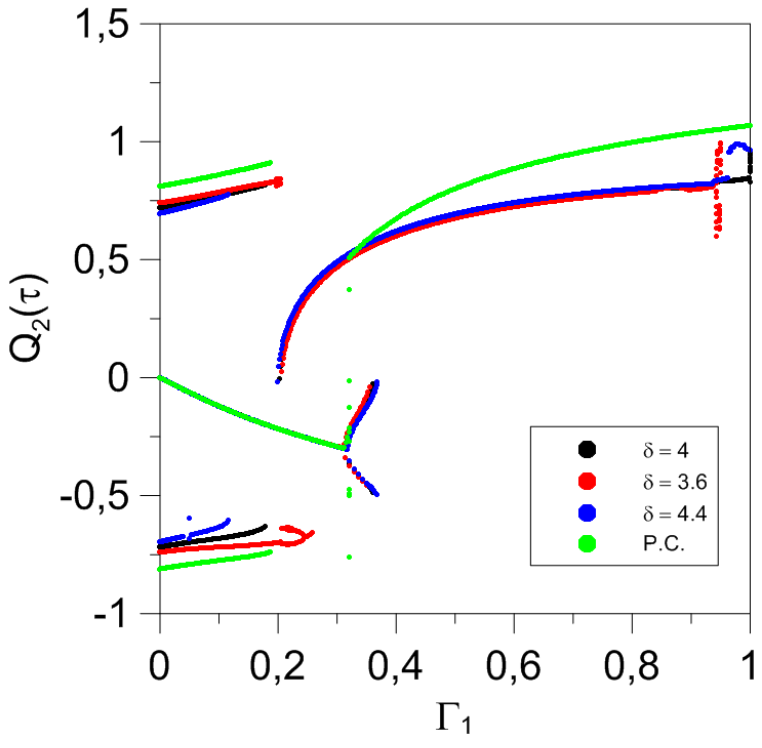

(b)

Fonte: Autores, 2020.

Na bifurcação do tipo Swallowtail o parâmetro de controle é $\alpha$, na Figura 6 é ilustrado os diagramas de bifurcação para o caso determinísticos e não determinístico do problema. Assim como as outras análises estocásticas do trabalho, serão considerados os seis primeiros termos do polinômio de Legendre-Caos da eq. (15) para a construção do diagrama de bifurcação para o caso não determinístico, representado pela cor verde. Já os diagramas de bifurcação para o parâmetro nominal e para as incertezas, estão representados pelas mesmas cores relacionadas anteriormente na Figura 3. Para a validação do método é necessário que a bifurcação não 
determinística represente a variabilidade do parâmetro $\alpha$ no intervalo $-3.3<\alpha<-2.7$, compreendido entre as curvas em azul e em vermelho, respectivamente.

Analisando a Figura 6, observa-se que para a bifurcação do tipo Swallowtail, o diagrama de bifurcação para o caso estocástico é mais alterado com a incorporação da variância das amostras na resposta no tempo, ele se comporta como a média das respostas somente em um pequeno trecho inicial da bifurcação, quando $\Gamma_{1}<0.04$. Tendo um comportamento diferente desse trecho inicial, à medida que se incrementa $\Gamma_{1}$. Observa-se que nesse trecho inicial $\left(\Gamma_{1}<\right.$ 0.04), o diagrama não determinístico apresenta duas soluções periódicas estáveis para o mesmo valor de $\Gamma_{1}$ assim como as bifurcações do parâmetro nominal (preto) e das incertezas em $\alpha$ (azul e vermelha). Cabe ressaltar que na região onde a incerteza de $\alpha=-2.7$ apresenta uma solução caótica, o diagrama de bifurcação regido pelo polinômio de caos, apresenta duas soluções estáveis para o mesmo valor de $\Gamma_{1}$, como ilustrado na Figura 6.

Figura 5 - Resposta no tempo não determinística para a bifurcação do tipo Butterfly, com incerteza em $\beta: \Gamma_{1}=0.05$ (a) $\mathrm{V}_{1}(\mathrm{t})$ e (a) $\mathrm{V}_{2}(\mathrm{t}) ; \Gamma_{1}=0.33$ (c) $\mathrm{V}_{1}(\mathrm{t})$ e (d) $\mathrm{V}_{2}(\mathrm{t}) .(\Omega=1.1)$

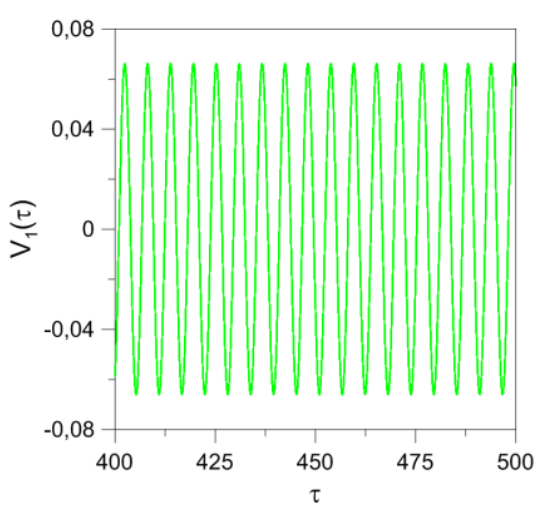

(a)

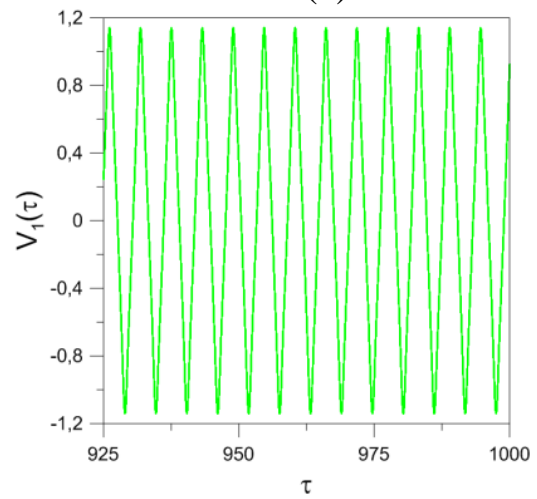

(c)

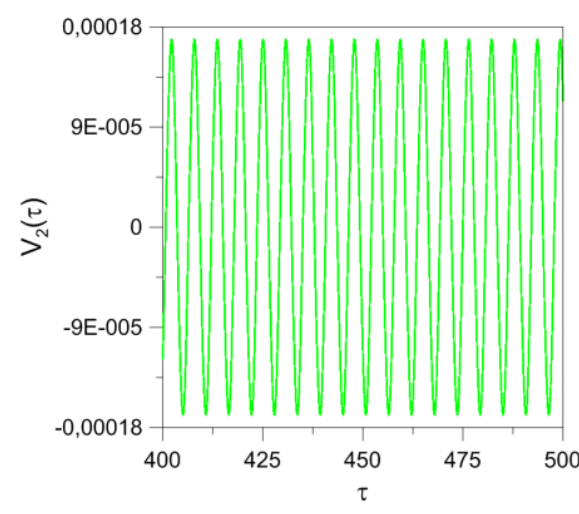

(b)

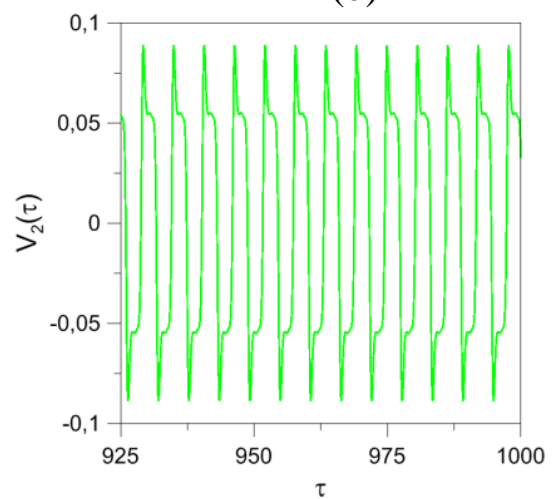

(d)

Fonte: Autores, 2020. 
Figura 6 - Diagramas de bifurcação determinístico e não determinístico do tipo Swallowtail, com incerteza em $\alpha .(\Omega=1.1, \lambda=4$, e $\xi=0.1)$.

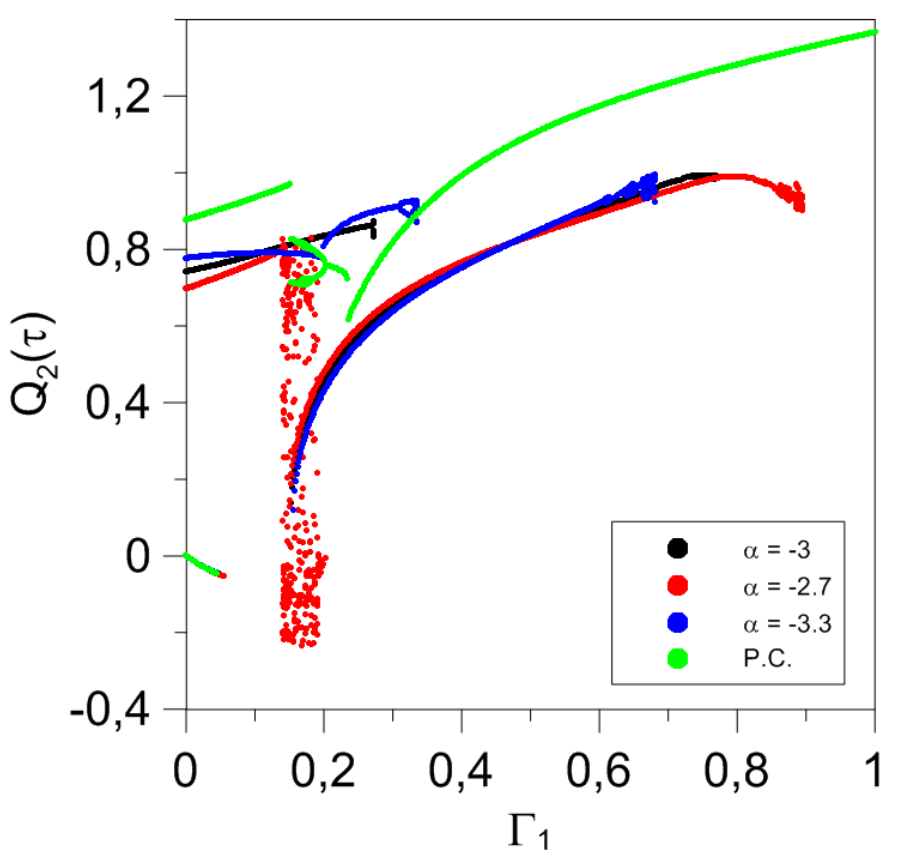

Fonte: Autores, 2020.

Assim como a bifurcação do tipo Butterfly, Figura 4, a bifurcação do tipo Swallowtail, Figura 6, possui um comportamento análogo em relação as suas bifurcações estocásticas. As mesmas apresentam incorporação à análise do problema a variância das amostras na resposta no tempo do sistema.

Na Figura 7 está representada a resposta no tempo estocástica, para a bifurcação do tipo Swallowtail incerteza em $\alpha$, para $\Gamma_{1}=0.04$ e 0.65 .

Onde pode ser observado que para o primeiro caso a variância é pequena, levando a resposta não determinística do sistema a se comportar com a média das respostas determinísticas, já para o segundo caso, a variância é o suficiente para modificar a resposta. 
Figura 7 - Resposta no tempo não determinística para a bifurcação do tipo Swallowtail, com incerteza em $\alpha: \Gamma_{1}=0.04$ (a) $\mathrm{V}_{1}(\mathrm{t})$ e (a) $\mathrm{V}_{2}(\mathrm{t}) ; \Gamma_{1}=0.65$ (c) $\mathrm{V}_{1}(\mathrm{t})$ e (d) $\mathrm{V}_{2}(\mathrm{t}) .(\Omega=1.1)$.

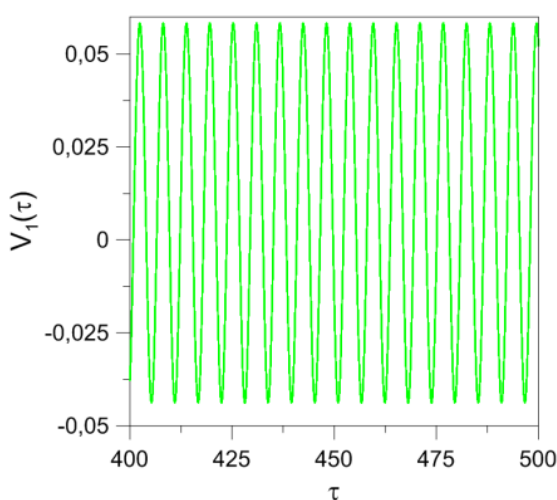

(a)

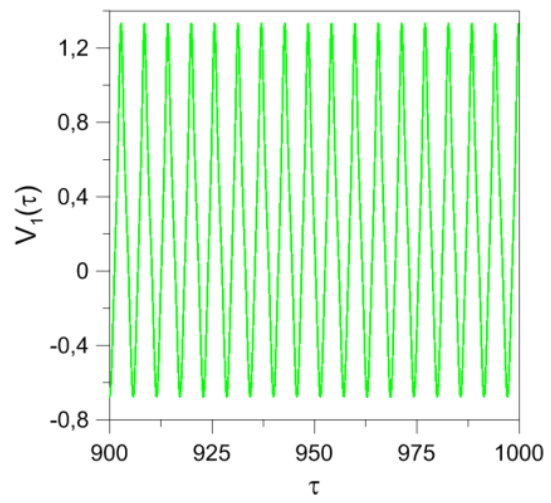

(c)

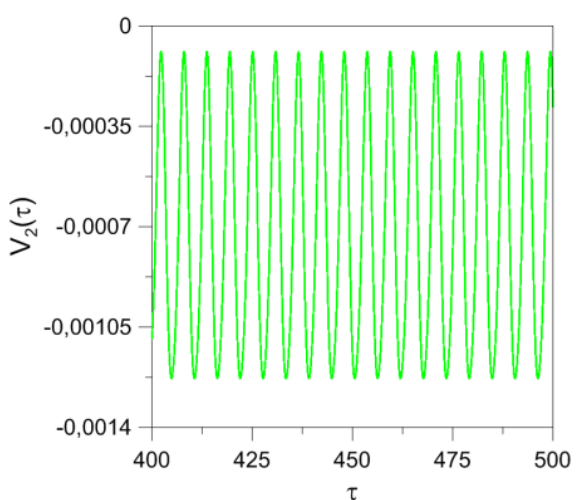

(b)

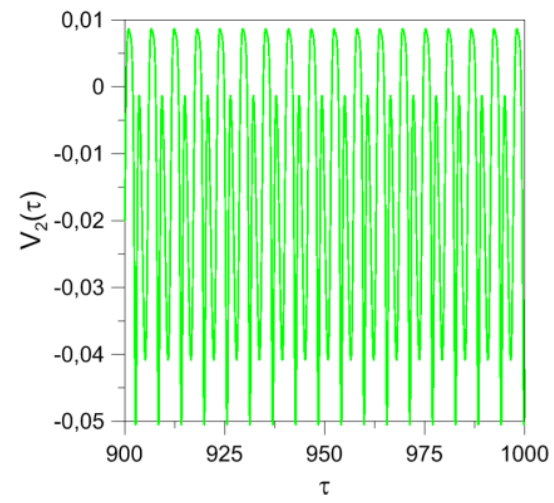

(d)

Fonte: Autores, 2020.

Para avaliar a periodicidade das soluções periódicas, ou dos movimentos caóticos, apresentados nos diagramas de bifurcação apresentados e, observando planos fases das respostas dinâmicas forçadas do modelo discreto estudado. Deve-se ficar atendo a sensibilidade das condições iniciais, pois, em uma integração no tempo com condições iniciais praticamente idênticas, ou seja, com pequenas variações em aproximação, podem divergir exponencialmente quando o movimento é caótico (Millon, 1991).

Analisa-se a resposta no espaço fase para os dois tipos de bifurcação: Butterfly e Swallowtail, com as suas respectivas incertezas nos parâmetros. Deve-se observar quando o movimento for periódico, ou seja, a órbita representada no plano fase fornece uma curva fechada com um número de seções de Poincarè, ou quando o movimento for caótico, com órbitas que nunca se repetem, apresentando um número indeterminado de seções de Poincarè. 
Figura 8 - Planos fases e seções de Poincarè para vibrações forçadas amortecidas do tipo Butterfly, para os casos determinísticos e não determinísticos com incerteza em $\beta .(\Omega=1.1, \delta=4, \lambda=4$ e $\xi=0.1)$.

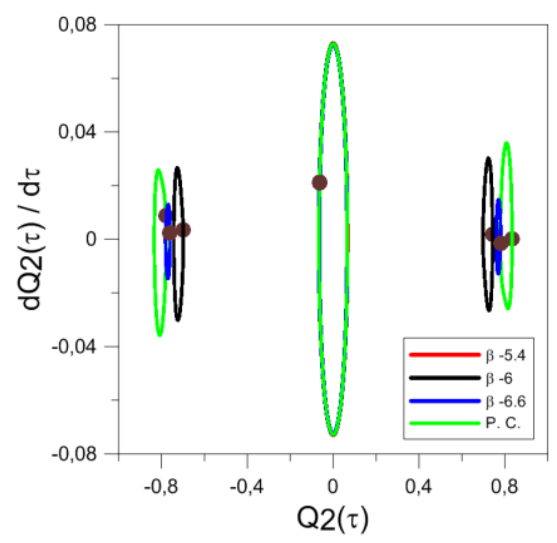

(a) $\Gamma_{1}=0.05$

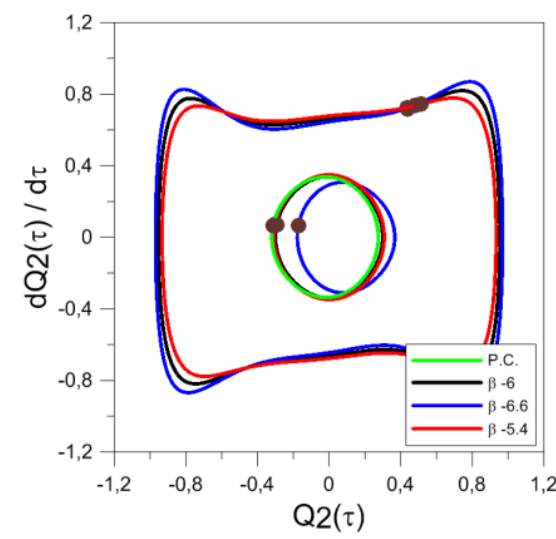

(b) $\Gamma_{1}=0.30$

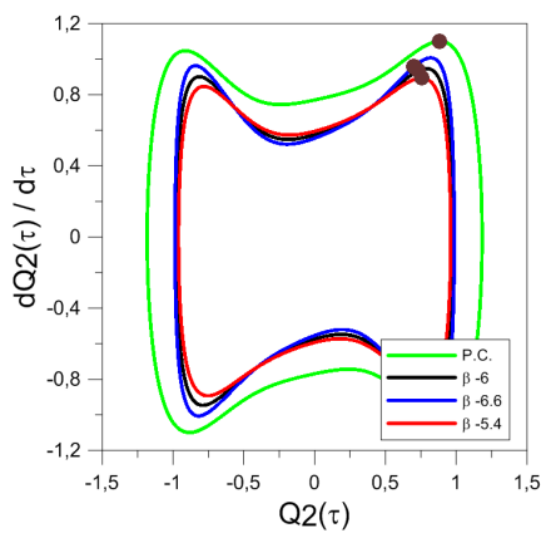

(c) $\Gamma_{1}=0.60$

Fonte: Autores, 2020.

Cabe ressaltar que os planos fases e as respostas no tempo, para os dois tipos de bifurcação analisados neste trabalho, foram obtidos assim como as análises anteriores para determinados valores de $\Gamma_{1}$, um pré-carregamento $\overline{P_{0}}=0.5$, parâmetro de frequência $\Omega=1.1$ e considerando uma taxa de amortecimento $\xi=0.1$. Os padrões em relações as cores de relaciona cada valor de incerteza também foram mantidos.

A Figura 8 mostra as seções de Poincarè, dadas pelos pontos marrons, e a resposta do plano fase analisadas para as vibrações forçadas amortecidas do tipo Butterfly para os casos determinísticos e não determinísticos com incerteza em $\beta$. Como pode ser observado, no estado permanente a resposta obtida para os planos fases tanto para os casos determinísticos quanto para os não determinísticos, para os três valores de $\Gamma_{1}$, estão em consonância com os diagramas de bifurcações da Figura 4a.

Na Figura 8a é ilustrado as seções de Poincarè e as respostas do plano fase para $\Gamma_{1}=0.05$, apresentando três planos fase com periodicidade unitária para o parâmetro nominal $\beta=-6$ (preto), $\beta=-6.6$ (azul), para $\beta=-5.4$ (vermelho) e para o polinômio de caos (verde), todas as órbitas apresentam um plano fase com periodicidade unitária, sendo possível observar que a resposta não determinística no vale potencial pré-flambagem converge para a média entre os valores analisados. Para as respostas presentes no vale pós-flambagem, a resposta não determinística da Figura 8a converge para limites superiores ou inferiores das órbitas determinísticas avaliadas. O comportamento da resposta estocásticas pode ser observado também na Figura 8c, onde o seu plano fase e seções de Poincarè envolve os demais planos fase dos casos determinísticos, atingindo assim valores maiores que os limites impostos pelas 
incertezas, comportamento que se explica devido a incorporação da variância das amostras na resposta no tempo do sistema. A partir das Figura 8 observa-se que quanto maior são os valores de $\Gamma_{1}$, o que propicia vibrações de grande amplitude entre os vales pré e pósflambagem, a resposta média do polinômio de caos não estará entre os limites do parâmetro analisado, prescrevendo órbitas com valores superiores aos valores determinísticos por conta da consideração da variabilidade que o método impõe e por conta da variabilidade de soluções que coexistem simultaneamente.

As análises dos planos fases e seções de Poincarè para as vibrações forçadas amortecidas do tipo Swallowtail, para os casos determinísticos e não determinísticos com incerteza em $\alpha$, para determinados valores de $\Gamma_{1}$ são apresentados na Figura 9. As relações de cores para os diferentes valores de $\alpha$ foram mantidas de acordo com a Figura 6. Como pode se analisar, no estado permanente, a resposta obtida para os planos fases tanto para os casos determinísticos quanto para os não determinísticos estão em consonância com os diagramas de bifurcações da Figura 6.

Figura 9 - Planos fases e seções de Poincarè para vibrações forçadas amortecidas do tipo Swallowtail, para os casos determinísticos e não determinísticos com incerteza em $\alpha .(\Omega=1.1, \lambda=4 \mathrm{e} \xi=0.1)$.

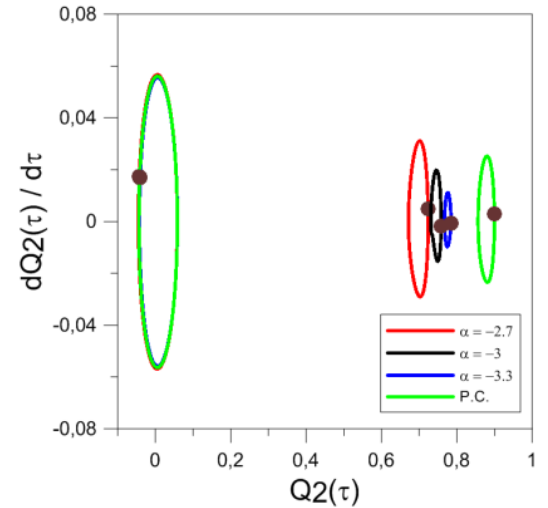

(a) $\Gamma_{1}=0.04$

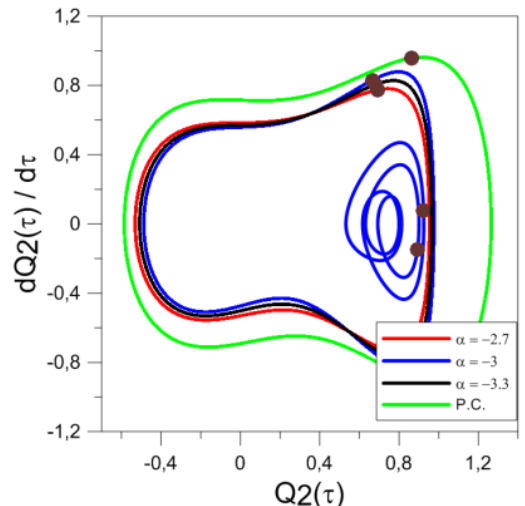

(b) $\Gamma_{1}=0.32$

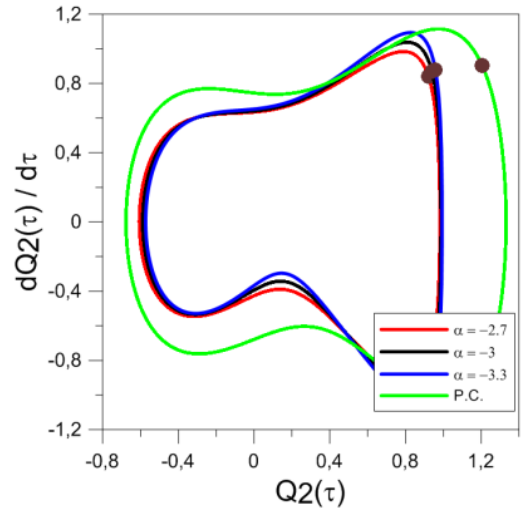

(c) $\Gamma_{1}=0.60$

Fonte: Autores, 2020.

Para $\Gamma_{1}=0.04$, Figura 9a, os resultados da seção de Poincarè e plano fase apresentam duas órbitas com periodicidade unitária, tanto para os casos determinísticos quanto para o não determinístico. Cabe ressaltar que a resposta estocástica se comporta com uma média dos resultados apenas para um plano fase, solução no vale pré-flambagem dado na Figura 9a. Para 
o plano fase das soluções do vale potencial pós-flambagem, os planos fases se comportam como um resultado limite, comportamento já esperando quando se analisa a Figura 6. Assim como na análise das respostas no tempo no caso não determinístico para a bifurcação do tipo Butterfly, a do tipo Swallowtail se comporta com valores limites à medida que se incrementa a amplitude a excitação harmônica, Figs. 9b e 9c, onde a órbita do plano fase do caso não determinístico envolve as órbitas dos casos determinísticos.

Foram obtidas as bacias de atração dinâmica através da equação (17), para os dois tipos de bifurcação avaliados neste trabalho e considerando-se as suas respectivas incertezas nos parâmetros determinísticos. Para o traçado das bacias de atração, foi utilizado o método de Runge-Kutta de quarta ordem para calcular o atrator de cada ponto pertencente a um plano que varia os deslocamentos e a velocidade do sistema discreto de [-1,1] e [-3,3], respectivamente. Os eixos horizontais simbolizam a variação da condição inicial de deslocamento $Q_{2}(\tau)$ e enquanto os eixos verticais varia-se a condição inicial de velocidade, $\mathrm{dQ}_{2}(\tau) / \mathrm{d} \tau$. Serão analisadas as bacias dos dois tipos de bifurcação: Butterfly e Swallowtail, para determinados valores de $\Gamma_{1}$ e um pré-carregamento $\overline{P_{0}}=0.5$, mantendo-se a frequência de excitação, $\Omega$, constante no valor de 1.1 , e considerando uma taxa de amortecimento $\xi=$ 0.1 .

Para os dois tipos de bifurcação analisados: Butterfly e Swallowtail, não se obteve as bacias de atração para o caso não determinístico por entender que as mesmas não fariam sentindo em uma comparação com os casos determinísticos, pois na análise não determinística se obtêm médias e variâncias enquanto que nas análises determinísticas se obtêm deslocamentos e velocidades.

A bifurcação do tipo Butterfly com o nível de carregamento estático igual 0.5, apresentam três soluções periódicas estáveis, sendo uma delas trivial, e duas soluções instáveis, independentemente de ser avaliada com parâmetro nominal ou com as incertezas em $\beta$ ou $\delta$. Na Figura 10 estão ilustradas as bacias de atração dinâmicas para este mesmo précarregamento, considerando-se as incertezas no parâmetro $\beta$, além de se avaliar várias amplitudes da excitação harmônica. Visando uma análise mais clara do comportamento das bacias de atração, dividiu-se os diagramas de bifurcações em quatro Grupos, como pode ser observado na Figura 2a. Os Grupos I e II são as soluções que convergem para o atrator direito (AD) nas bacias de atração e serão representados respectivamente pelas cores vermelho e amarelo. Já o Grupo III representa as soluções que convergem para o atrator central (AC), 
Research, Society and Development, v. 9, n. 7, e424973763, 2020

(CC BY 4.0) | ISSN 2525-3409 | DOI: http://dx.doi.org/10.33448/rsd-v9i7.3763

sendo representadas pela cor preta. O Grupo IV será representado nas bacias de atração pela cor azul para indicar soluções que convergem para o atrator esquerdo (AE).

Figura 10 - Bacias de atração dinâmicas para a bifurcação do tipo Butterfly, com incerteza no parâmetro $\beta$, para diferentes valores de $\Gamma_{1} .(\Omega=1.1, \delta=4, \lambda=4$ e $\xi=0.1)$.

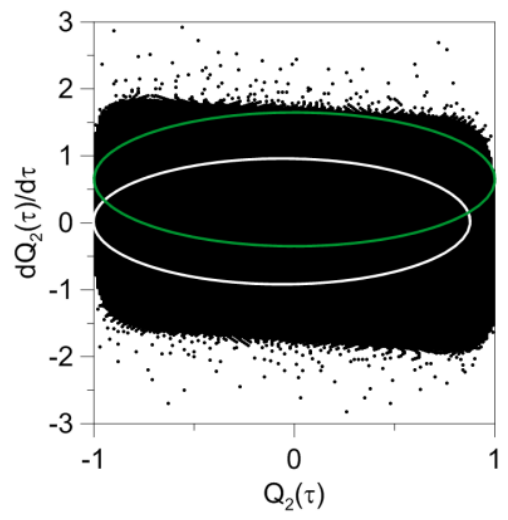

(a) $\Gamma_{1}=0.05 ; \beta=-5.4$

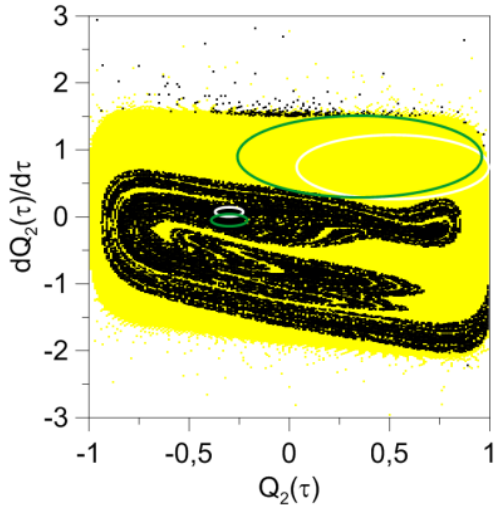

(d) $\Gamma_{1}=0.30 ; \beta=-5.4$

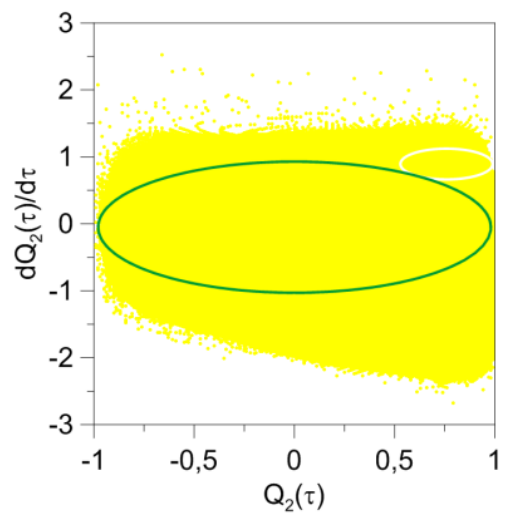

(g) $\Gamma_{1}=0.60 ; \beta=-5.4$

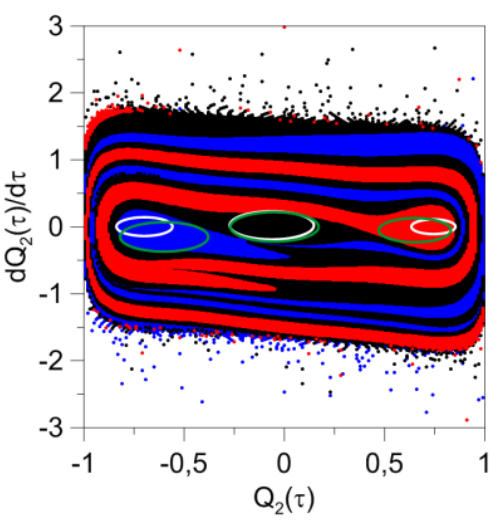

(b) $\Gamma_{1}=0.05 ; \beta=-6$

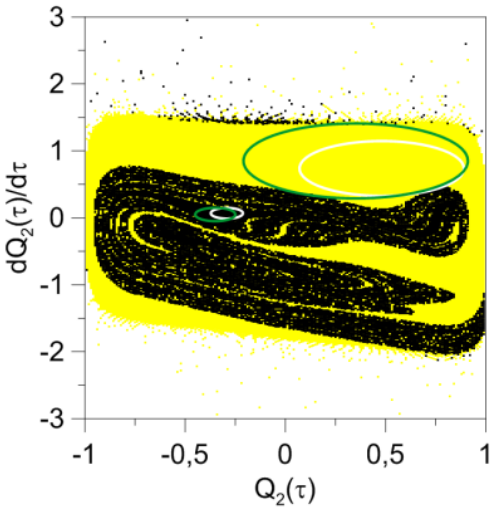

(e) $\Gamma_{1}=0.30 ; \beta=-6$

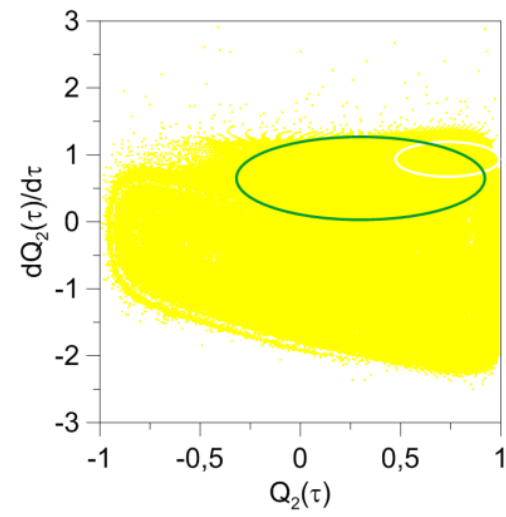

(h) $\Gamma_{1}=0.60 ; \beta=-6$

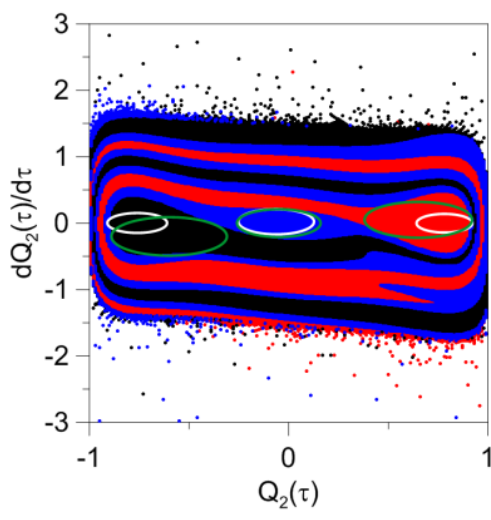

(c) $\Gamma_{1}=0.05 ; \beta=-6.6$

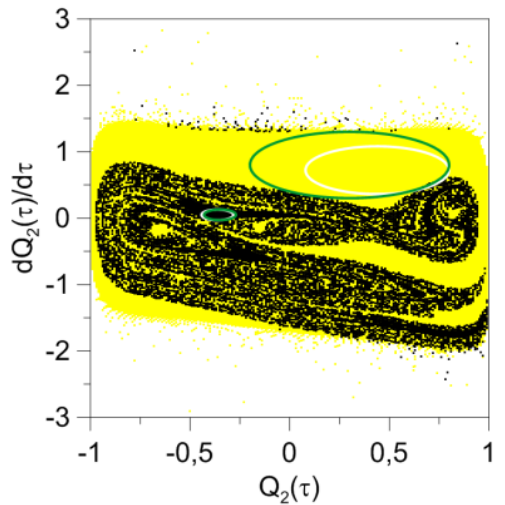

(f) $\Gamma_{1}=0.30 ; \beta=-6.6$

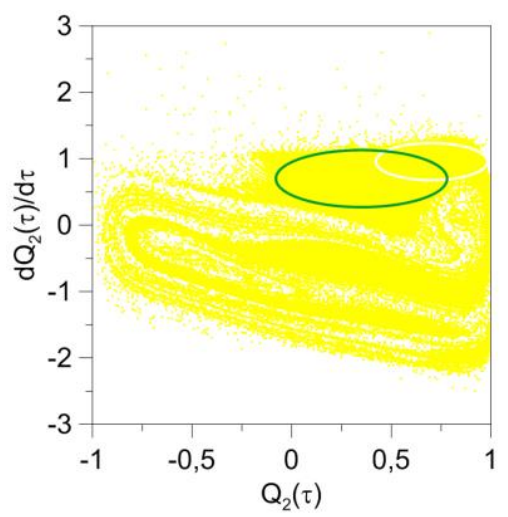

(i) $\Gamma_{1}=0.60 ; \beta=-6.6$

Fonte: Autores, 2020. 
Nota-se, a partir da Figura 10, que à medida se que aumenta a amplitude da excitação harmônica, independentemente do valor da incerteza, maior é a tendência das condições iniciais da bacia de atração se estabilizar no foco direito (região de cor amarela) ou central (região de cor preta), minimizando a região do foco esquerdo (região de cor azul). Nota-se também que com o incremento de $\Gamma_{1}$ há uma redução no número de soluções. Nas Figs. $10(\mathrm{~g})$ - (i) pode-se notar a redução significativa na área das bacias de atração. A região de cor branca das bacias de atração corresponde às condições iniciais as quais as soluções tendem ao infinito.

Juntamente com as bacias de atração foram analisados os fatores de integridades locais e globais do sistema com bifurcação Butterfly com a incerteza em $\beta$. Os fatores de integridades estão apresentados pelas regiões delimitadas pelos círculos nas bacias de atração da Figura 10. O fator de integridade local está representado pela circunferência de cor branca, já o fator de integridade global está representado pela circunferência de cor verde.

Com o objetivo de analisar o comportamento dos fatores de FIL e FIG como um todo, unificou-se a Figura 11, que reúne todos os fatores de integridade, locais e globais, para o parâmetro nominal $\beta=-6$, mantendo-se na Figura 11 as relações de cores designadas para cada Grupo da Figura 2a.

Figura 11 - Fatores de integridade para a bifurcação do tipo Butterfly, para o parâmetro nominal $\beta=$ 6 referente aos Grupos da Figura 2a: Fator de integridade (a) Local (b) Global.

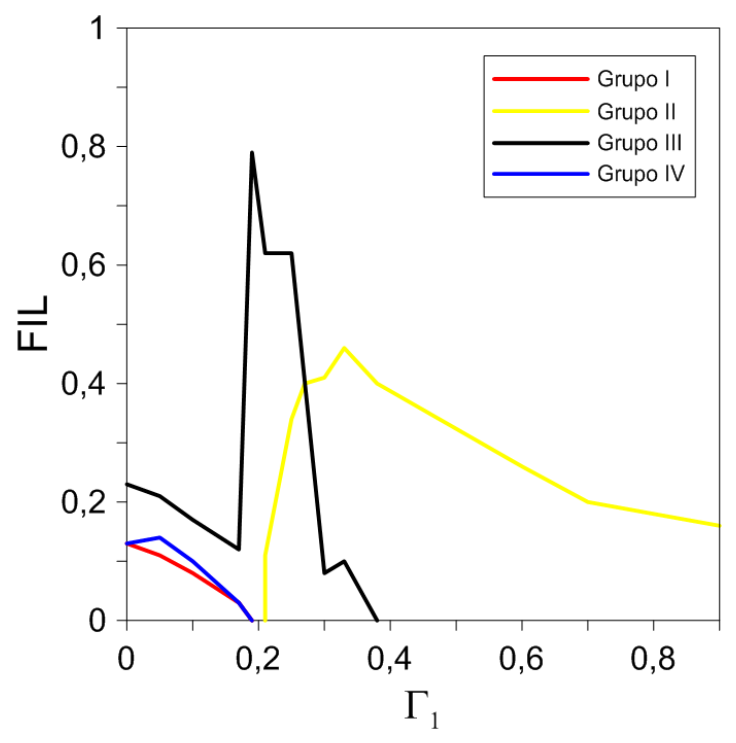

(a)

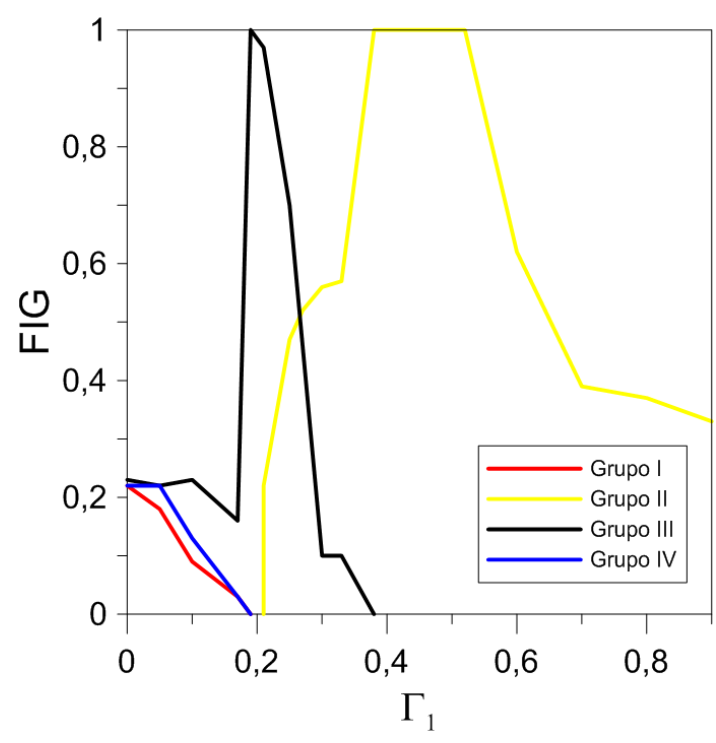

(b)

Fonte: Autores, 2020. 
A partir da Figura 11 pode-se observar de forma geral que tanto os FILs, quanto os FIGs diminuem com o incremento da amplitude da carga harmônica $\Gamma_{1}$, e possuem os maiores valores de raio, quando as soluções convergem para o Grupo III (cor preta) para qualquer valor de $\Gamma_{1}<0.27$. Cabe ressaltar que o maior valor para o FIL e FIG acontece no Grupo III, quando $\Gamma_{1}=0.19$, já que para esse valor acabam os FILs e FIGs para os Grupos I e IV, representados respectivamente pelas cores vermelha e azul. Os valores dos FIL e FIG do Grupo II, representado pela cor amarela, começam a surgir quando terminam os FILs e FIGs do Grupo I e IV. A partir das Figuras 2a e 11 pode-se observar que nas regiões onde acabam as soluções periódicas estáveis para um determinado Grupo, começa as soluções periódicas estáveis em outro Grupo. Isso reflete nos fatores de integridade, enquanto os FIL e FIG de um Grupo vai diminuindo os de um outro Grupo vai aumentando, esse comportamento pode ser observado na Figura 11 entre o Grupo II e III, onde à medida que os FIL e FIG do primeiro aumentam os do segundo diminuem.

\section{Considerações Finais}

Neste trabalho, avaliou-se, para um sistema mecânico susceptível a bifurcação, a influência de incertezas nos parâmetros de rigidez do sistema nos diagramas de bifurcação dinâmico, bem como a aplicabilidade do polinômio de caos para estudo das aleatoriedades deste sistema. Quanto a rigidez do sistema discreto, dependendo dos valores iniciais da mola não linear é possível obter repostas pós-críticas com diferentes topologias, que neste trabalho foram consideradas: a bifurcação simétrica instável do tipo Butterfly e a bifurcação assimétrica do tipo Swallowtail.

Foram traçados os diagramas de bifurcação dinâmico para as respostas determinísticas e não determinísticas paras os dois tipos de bifurcação com seus respectivos parâmetros nominais e com incertezas. Podendo concluir a partir dos resultados que os diagramas de bifurcação do tipo Butterfly, para a análise determinística dinâmica, sofre maior modificação em relação as incertezas quando ela é avaliada no parâmetro $\beta$. Dependendo da incerteza em $\beta$ pode surgir mais soluções periódicas estáveis quando comparado as soluções obtidas para o parâmetro nominal.

Já as incertezas impostas no parâmetro $\delta$ pouco modifica o comportamento do diagrama de bifurcação, tendo assim um comportamento análogo ao parâmetro nominal. Assim como a bifurcação do tipo Butterfly, a Swallowtail também é modificada de forma 
significativa em relação a variação das incertezas, quando se analisa seus diagramas de bifurcação determinístico. Dependendo do valor que se insere a incerteza pode acontecer um aumento no número de soluções estáveis, mas dependendo do valor também pode gerar soluções caóticas.

A partir das análises não determinísticas conclui-se que a aplicabilidade do polinômio de caos fornece a média das respostas apenas para as soluções do vale pré-flambagem. Para os diagramas de bifurcação estocásticos obtidos com o polinômio de Legendre-Caos, independentemente da bifurcação analisada, os resultados obtidos se comportaram como a média dos resultados limites apenas para pequenos valores de $\Gamma_{1}$ ou para as soluções presentes no vale potencial pré-flambagem.

Concluindo que esse comportamento acontece porque a técnica do polinômio de caos incorpora à análise do problema a variância das amostras na resposta no tempo do sistema, logo para incrementos grandes de $\Gamma_{1}$, a variância é suficiente para modificar a resposta não determinística do sistema. Cabendo assim para trabalhos futuros estudar a aplicabilidade do polinômio de caos para problemas dinâmicos não-lineares, de modo que a variabilidade das amostras na resposta no tempo possa ser controlada ou prescrita.

\section{Referências}

Cedolin, L., Bazant, Z. P., 2003. Stability of Structures, Grupo Dover Science, 1014p.

Gonçalves, P. B., Silva, F. M. A., Rega, G., Lenci, S., 2011. Global dynamics and integrity of a two-dof model of a parametrically excited cylindrical shell. Nonlinear Dynamics, 63 (1), 6182.

Hoff, A., 2014. Estruturas de bifurcação em sistemas dinâmicos quadridimensionais.

Dissertação (Mestrado em Física) - Departamento de Física, Universidade do Estado de Santa Catarina, Centro de Ciências Tecnológicas, Joinville, 65 p.

Lenci, S., Rega, G., 2003. Optimal control of nonregular dynamics in a Duffing oscillator. Nonlinear Dynamics. 33 (1), 71-86.

Millon, S. L. J.,1991. Técnicas gráficas e computacionais para a análise de oscilações não lineares e caos em sistemas estruturais suscetíveis à flambagem. Dissertação (Mestrado em 
Engenharia Civil: Estruturas) - Departamento de Engenharia Civil, Pontifica Universidade Católica do Rio de Janeiro, Rio de Janeiro, 232p.

Nayfeh, AH, Balachandran, B. (1995). Applied nonlinear dynamics: analytical, computational, and experimental methods ( $1^{\mathrm{a}}$ ed.). Editora: John Wiley Andamp, 69p.

Rega, G, Lenci, S. (2005). Identifying, evaluating and controlling dynamical integrity measures in non-linear mechanical oscillators. Nonlinear Anal. 63 (2), 902-914.

Rega, G, Lenci, S. (2008). Dynamical integrity and control of nonlinear mechanical oscillators. J. Vib. 14 (1), 159-179.

Silva, FMA, Brazão, AF, Gonçalves, PB, 2015. "Influence of physical and geometrical uncertainties in the parametric instability load of an axially excited cylindrical shell”. Mathematical Problems in Engineering, 35 (2), 151-175.

Silva, FMA, Gonçalves, PB. (2011).The influence of uncertainties and random noise on the dynamic integrity analysis of a system liable to unstable buckling. Nonlinear Dynamics, 66 (1), 303-333.

Thompson, JMT. (1989). Chaotic phenomena triggering the escape from a potential well. Proc. R. Soc. Lond. 421,195-225.

Xiu, D, Karniadakis, GE. (2002). The Wiener-Askey polynomial chaos for stochastic differential equations. SIAM Journal on Scientific Computing, 24 (2), 619-644.

\section{Porcentagem de contribuição de cada autor no manuscrito}

Michael Dowglas de Gois Silva- 33,33\%

Fabio Roberto Chavarette- 33,33\%

Rodrigo Francisco Borges Lourenço-33,33\% 